\title{
P-th moment and almost sure stability of stochastic switched nonlinear systems
}

*Correspondence: gaocx0471@163.com School of Mathematical Sciences, Inner Mongolia University, Hohhot 010021 People's Republic of China

\begin{abstract}
This paper mainly tends to utilize $\psi$-type function to investigate p-th moment and almost sure stability for a class of stochastic switched nonlinear systems. Based on the multiple Lyapunov functions approach, some sufficient conditions are derived to check the stability criteria of stochastic switched nonlinear systems. One numerical example is provided to demonstrate the effectiveness of the proposed results.
\end{abstract}

Keywords: Stochastic switched nonlinear system, P-th moment stability, Almost sure stability, Multiple Lyapunov functions approach

Mathematics Subject Classification: Primary 60H10, 34D20; Secondary 93C30

\section{Background}

Switched system is an indispensable class of hybrid dynamical systems, which is composed of a family of subsystems and a rule that orchestrates the switching among them. Yet, there exist stochastic effects in the practical systems such as physics, biology, information science and economic. (for instance, see Xie and Wang 2003; Zhang and Nie 2004; Goetz and Hritonenko 2008). Over the previous few decades, stochastic switched systems have received much attention due to their potential applications in many fields, such as the control of mechanical systems, automotive industry, aircraft and air traffic control, chemical and electrical engineering, etc., see e.g. Krystul (2001), Øksendal (2005).

As is well known, stability is one of the major issues in the study of control theory. In particular, the existence, uniqueness and stability of solutions for stochastic systems are investigated in Mao (1997), Hu et al. (2008), Zhang and Chen (2004) and the stability results of switched systems are given in $\mathrm{Hu}$ et al. (1999) and Aleksandrov et al. (2011). Some exponential stability, almost sure exponential stability and p-th moment stability criteria are obtained for stochastic systems in Mao (1994), Khasminskii (1980), Shen and Wang (2009). Recently, some efforts have been made to extend the stability results from stochastic systems to stochastic switched systems (Feng and Zhang 2006; Feng et al. 2011; Chatterjee and Liberzon 2004; Filipovic 2009; Ai and Zong 2014). In Branicky (1998), Dimarogonas and Kyriakopoulos (2004) and Chatterjee and Liberzon (2006) the investigators utilize multiple Lyapunov functions approach to study the stability of stochastic switched systems. P-th moment exponential stability of stochastic switched

(c) The Author(s) 2016. This article is distributed under the terms of the Creative Commons Attribution 4.0 International License (http://creativecommons.org/licenses/by/4.0/), which permits unrestricted use, distribution, and reproduction in any medium, provided you give appropriate credit to the original author(s) and the source, provide a link to the Creative Commons license, and indicate if changes were made. 
systems is investigated in Zhang et al. (2014) and Wu et al. (2013) and almost sure exponential stability of stochastic switched systems is researched in Cong et al. (2011). Although the stability of stochastic switched systems has stirred some initial research interest, there still leaves much room for further investigations to reduce the possible conservations.

For instance, in Hu et al. (2008), Wu and $\mathrm{Hu}$ (2012) and Pavlovic and Jankovic (2012), the researchers introduce $\psi$-type function and investigate p-th moment and almost surely $\psi^{\gamma}$ stability for stochastic nonlinear systems. Since $\psi^{\gamma}$ stability contains exponential stability and polynomial stability, it has a wide applicability. However, there are few research results about p-th moment and almost sure $\psi^{\gamma}$ stability for stochastic switched nonlinear systems.

In this paper, we attempt to investigate p-th moment and almost sure $\psi^{\gamma}$ stability of stochastic switched nonlinear systems. Since the switching behavior exists among stochastic switched systems, the stability of subsystems does not guarantee the stability of the whole system. By the aid of the semi-martingale convergence theorem, we obtain the p-th moment $\psi^{\gamma}$ stability of stochastic switched nonlinear systems. In order to establish the criterion on almost surely $\psi^{\gamma}$ stable of stochastic switched nonlinear systems, we improve the exponential martingale inequality in this paper.

The paper is organized as follows. Firstly, the problem formulations, definitions of $\psi^{\gamma}$ stability and some lemmas are given in "Preliminaries" section. In third section, the main results on p-th moment $\psi^{\gamma}$ stability and almost surely $\psi^{\gamma}$ stability of stochastic switched nonlinear systems are obtained using multiple Lyapunov functions. An example is presented to illustrate the main results in "Examples" section . In the last section the conclusions are given.

\section{Preliminaries}

Throughout this paper, unless otherwise specified, we let $R^{n}$ be the n-dimensional Euclidean space; $R_{+}$is the set of all non-negative real numbers; $R^{n \times m}$ denotes the $n \times m$ real matrix space; $|\cdot|$ denotes the standard Euclidean norm for vectors; $\mathcal{C}^{1,2}\left(R_{+} \times R^{n}\right)$ denotes the family of all non-negative functions $V(t, x(t))$ on $R_{+} \times R^{n}$ which are twice continuously differentiable in $x$ and once in $t ; L^{p}\left(\Omega, R^{n}\right)$ denotes the family of $R^{n}$ valued random variables $\xi$ with $E|\xi|^{p}<\infty ; a \bigvee b$ denotes the maximum of a and b; $\mathcal{L}^{p}\left([a, b], R^{n}\right)$ denotes the family of $R^{n}$-valued $\mathcal{F}_{t}$-adapted processes $\{f(t)\}_{a \leq t \leq b}$ such that $\int_{a}^{b}|f(t)|^{p} d t<\infty$ a.s.; $P(\cdot)$ means the probability of a stochastic process; $E[\cdot]$ means the expectation of a stochastic process; $\mathbb{N}=1,2, \ldots, N$ is a discrete index set, where $N$ is a finite positive integer.

Consider a family of stochastic switched nonlinear systems described by

$$
\left\{\begin{array}{l}
d x(t)=f_{\sigma(t)}(t, x(t)) d t+g_{\sigma(t)}(t, x(t)) d w(t) \\
x\left(t_{0}\right)=x_{0}, \quad t_{0}=0
\end{array}\right.
$$

where $\sigma(t):\left[t_{0}, \infty\right) \rightarrow N$ is the switching signal, let $\left\{t_{1}<t_{2}<\cdots<t_{k}<\cdots\right\}$ be a switching sequence and the $i_{k}$-th subsystem is active at time interval $\left[t_{k}, t_{k+1}\right]$, where $i_{k}$ is the switching instant, $i_{k} \in \mathcal{N}, k=0,1,2, \ldots$ System (1) is consisted with many stochastic subsystems $d x(t)=f_{i}(t, x(t)) d t+g_{i}(t, x(t)) d w(t)$ which are driven by 
switching signal $\sigma(t) . x(t) \in R^{n}$ is the state of the system, $w(t)$ is an m-dimensional Brownian motion defined on the complete probability space $\left(\Omega, \mathcal{F},\left\{\mathcal{F}_{t}\right\}, P\right)$, with filtration $\mathcal{F}_{t}$ satisfying the usual conditions (i.e. it is increasing and right continuous while $\mathcal{F}_{0}$ contains all P-null sets), functions $f: R_{+} \times R^{n} \rightarrow R^{n}, g: R_{+} \times R^{n} \rightarrow R^{n \times m}$ are locally Lipschitz in $x(t) \in R^{n}$ and piecewise continuous in $t$ for all $t \geq t_{0}$ and $f(t, 0)=0, g(t, 0)=0, t \in\left[t_{0}, \infty\right)$.

For the existence and uniqueness of the solution we impose an assumption (A):

Both $f_{i}(t, x(t))$ and $g_{i}(t, x(t))$ satisfy the Lipschitz condition and the linear growth condition. That is, there exist a group of constants $L_{i}>0$ such that

$$
\left|f_{i}(t, x)-f_{i}(t, y)\right|^{2} \vee\left|g_{i}(t, x)-g_{i}(t, y)\right|^{2} \leq L_{i}\|x-y\|^{2}
$$

For all $t \geq 0$, and $x, y \in R^{n}$, and, moreover, there is a group of constants $K_{i}>0$ such that

$$
\left|f_{i}(t, x)\right|^{2} \vee\left|g_{i}(t, x)\right|^{2} \leq K_{i}\left(1+\|x\|^{2}\right)
$$

For all $t \geq 0$, and $x \in R^{n}$.

The purpose of this paper is to investigate the pth moment and almost sure $\psi^{\gamma}$ stability of system (1), we first introduce some definitions as follows.

Definition 1 (Hu et al. 2008) $\psi(t): R_{+} \rightarrow(0,1]$ is said to be $\psi$-type function, if it satisfies the following conditions:

1. It is continuous and differentiable;

2. $\psi(0)=1$ and $\psi(t) \rightarrow 0$, as $t \rightarrow \infty$;

3. $\psi_{1}(t)=\psi^{\prime}(t) / \psi(t)<0$;

4. for any $t, s \geq 0, \psi(t+s) \geq \psi(t) \psi(s)$.

Definition 2 For $p>0$, the stochastic switched nonlinear system (1) is said to be $\mathrm{p}$-th moment $\psi^{\gamma}$ stable, if there exist positive constants $\beta, \gamma$ and function $\psi(\cdot)$ defined above, such that

$$
E\left|x\left(t, x_{0}\right)\right|^{p} \leq \beta\left|x_{0}\right|^{p} \psi^{\gamma}(t)
$$

when $p=2$, we say that it is $\psi^{\gamma}$ stable in mean square, when $\psi(t)=e^{-t}$, we say that it is p-th moment exponential stable, when $\psi(t)=(1+t)^{-1}$,we say that it is p-th moment polynomial stable.

Definition 3 Stochastic switched nonlinear systems (1) is said to be almost surely $\psi^{\gamma}$ stable, if there exist a positive constant $\gamma$ and function $\psi(\cdot)$ defined above, such that

$$
\limsup _{t \rightarrow \infty} \frac{\ln \left|x\left(t, x_{0}\right)\right|}{|\ln \psi(t)|} \leq-\gamma, \quad \text { a.s. }
$$

when $\psi(t)=e^{-t}$, we say that it is almost surely exponential stable, when $\psi(t)=(1+t)^{-1}$, we say that it is almost surely polynomial stable. 
Before giving some efficient lemmas, let us introduce Itô formula. For system (1), give any function $V(t, x) \in \mathcal{C}^{1,2}\left(R_{+} \times R^{n}\right)$ and define operators $d V(t, x)$ and $\mathcal{L} V(t, x)$ as follows.

$$
\begin{aligned}
& d V(t, x)=\mathcal{L} V(t, x) d t+V_{x}(t, x) g(t, x) d w(t) \\
& \mathcal{L} V(t, x)=V_{t}(t, x)+V_{x}(t, x) f(t, x)+\frac{1}{2} \operatorname{tr}\left[g^{T}(t, x) V_{x x}(t, x) g(t, x)\right]
\end{aligned}
$$

where

$V_{t}(t, x)=\frac{\partial V(t, x)}{\partial t}, V_{x}(t, x)=\left(\frac{\partial V(t, x)}{\partial x_{1}}, \ldots, \frac{\partial V(t, x)}{\partial x_{n}}\right), V_{x x}(t, x)=\left(\frac{\partial^{2} V(t, x)}{\partial x_{i} \partial x_{j}}\right)_{n \times n}$

Lemma 4 (Hu et al. 2008; Semi-martingale Convergence Theorem) Let $M(t)$ is a real value continuous local martingale, and $M(0)=0$, a.s., $\zeta$ is an $\mathcal{F}_{0}$ measurable non-negative random variable, if $X(t)$ is an $\mathcal{F}_{t}$ adapted continuous non-negative process and satisfies with

$$
X(t) \leq \zeta+M(t), \quad t \geq 0
$$

then, $E X(t) \leq E \zeta$, and $X(t)$ is bounded a.s., that is

$$
\limsup _{t \rightarrow \infty} X(t)<\infty \text {, a.s. }
$$

Lemma 5 (Mao 1997; Doob's Martingale Inequalities) Let $\left\{M_{t}\right\}_{t \geq 0}$ be an $R^{n}$-valued martingale. Let $[a, b]$ be a bounded interval in $R_{+}$.

1. if $p \geq 1$ and $M_{t} \in L^{p}\left(\Omega, R^{n}\right)$, then for all $c>0$,

$$
P\left(\sup _{a \leq t \leq b}\left|M_{t}(\omega)\right| \geq c\right) \leq c^{-p} E\left|M_{b}\right|^{p} .
$$

2. if $p>1$ and $M_{t} \in L^{p}\left(\Omega, R^{n}\right)$, then

$$
E\left(\sup _{a \leq t \leq b}\left|M_{t}\right|^{p}\right) \leq\left(\frac{p}{p-1}\right)^{p} E\left|M_{b}\right|^{p} .
$$

In order to establish the criterion on almost sure $\psi^{\gamma}$ stability of stochastic switched nonlinear systems, we need to modify the exponential martingale inequality as follows.

Lemma 6 (Exponential Martingale inequality) Let $g \in \mathcal{L}^{2}\left([0, T] \times R^{n}\right), \forall \varepsilon, \delta>0$, then

$$
P\left(\sup _{0 \leq t \leq T}\left[\int_{0}^{t} \frac{|\ln \psi(t)|}{t} g(s) d w(s)-\frac{\delta}{2} \int_{0}^{t} \frac{\ln ^{2} \psi(t)}{t^{2}}|g(s)|^{2} d s\right] \geq \varepsilon\right) \leq e^{-\delta \varepsilon} .
$$


Gu and Gao SpringerPlus (2016)5:2002

Page 5 of 13

Proof For any positive integer $n \geq 1$, define a stopping time:

$$
\tau_{n}=\inf \left\{t \geq 0:\left|\int_{0}^{t} \frac{|\ln \psi(t)|}{t} g(s) d w(s)\right|+\int_{0}^{t} \frac{\ln ^{2} \psi(t)}{t^{2}}|g(s)|^{2} d s>n\right\}
$$

and a process:

$$
y(t)=\delta \int_{0}^{t} I_{\left[0, \tau_{n}\right]}(s) \frac{|\ln \psi(t)|}{t} g(s) d w(s)-\frac{\delta^{2}}{2} \int_{0}^{t} I_{\left[0, \tau_{n}\right]}(s) \frac{\ln ^{2} \psi(t)}{t^{2}}|g(s)|^{2} d s,
$$

where $\left[0, \tau_{n}\right]$ is a stochastic time interval. When the set

$$
\left\{t \geq 0:\left|\int_{0}^{t} \frac{|\ln \psi(t)|}{t} g(s) d w(s)\right|+\int_{0}^{t} \frac{\ln ^{2} \psi(t)}{t^{2}}|g(s)|^{2} d s>n\right\}
$$

is empty, $\tau_{n}=\inf \phi=\infty$, obviously, $\tau_{n} \uparrow \infty$. By Ito formula, we have

$$
\begin{aligned}
e^{y(t)}= & 1+\int_{0}^{t} e^{y(s)}\left[\delta I_{\left[0, \tau_{n}\right]}(s) \frac{|\ln \psi(t)|}{t} g(s) d w(s)-\frac{\delta^{2}}{2} I_{\left[0, \tau_{n}\right]}(s) \frac{\ln ^{2} \psi(t)}{t^{2}}|g(s)|^{2} d s\right] \\
& +\frac{1}{2} \delta^{2} \int_{0}^{t} I_{\left[0, \tau_{n}\right]}(s) \frac{\ln ^{2} \psi(t)}{t^{2}}|g(s)|^{2} d s \\
= & 1+\delta \int_{0}^{t} e^{y(s)} I_{\left[0, \tau_{n}\right]}(s) \frac{|\ln \psi(t)|}{t} g(s) d w(s)
\end{aligned}
$$

That is, $e^{y(t)}$ is a non-negative martingale for all $t \geq 0, E\left[e^{y(t)}\right]=1$.

By the first part of Lemma 5 with $p=1$, we obtain

$$
P\left(\sup _{0 \leq t \leq T} y(t) \geq \delta \varepsilon\right)=P\left(\sup _{0 \leq t \leq T} e^{y(t)} \geq e^{\delta \varepsilon}\right) \leq e^{-\delta \varepsilon} E\left[e^{y(T)}\right]=e^{-\delta \varepsilon} .
$$

That is

$$
P\left(\sup _{0 \leq t \leq T}\left[\int_{0}^{t} I_{\left[0, \tau_{n}\right]}(s) \frac{|\ln \psi(t)|}{t} g(s) d w(s)-\frac{\delta}{2} \int_{0}^{t} I_{\left[0, \tau_{n}\right]}(s) \frac{\ln ^{2} \psi(t)}{t^{2}}|g(s)|^{2} d s\right] \geq \varepsilon\right) \leq e^{-\delta \varepsilon} .
$$

Let, $n \rightarrow \infty$, we have

$$
P\left(\sup _{0 \leq t \leq T}\left[\int_{0}^{t} \frac{|\ln \psi(t)|}{t} g(s) d w(s)-\frac{\delta}{2} \int_{0}^{t} \frac{\ln ^{2} \psi(t)}{t^{2}}|g(s)|^{2} d s\right] \geq \varepsilon\right) \leq e^{-\delta \varepsilon} .
$$

So, the proof is completed. 
Lemma 7 (Mao 1997; Borel-Cantelli's Lemma) If $\left\{A_{k}\right\} \subset \mathcal{F}$ and $\sum_{k=1}^{\infty} P\left(A_{k}\right)<\infty$, then

$$
P\left(\limsup _{k \rightarrow \infty} A_{k}\right)=0 .
$$

That is, there exist a set $\Omega_{o} \in \mathcal{F}$ with $P\left(\Omega_{o}\right)=1$ and an integer valued random variable $k_{o}$ such that for every $\omega \in \Omega_{o}$ we have $\omega \notin A_{k}$ whenever $k \geq k_{o}(\omega)$.

\section{Main results}

In this section, we shall tend to investigate a family of stochastic switched nonlinear systems by using multiple Lyapunov functions approach and give some sufficient conditions estimating the $\mathrm{p}$-th moment and almost surely $\psi^{\gamma}$ stable. Before giving the efficient theorems, we assume that the switching signal $\sigma(t)$ is right continuous.

Let us turn our attention to system (1) and give some sufficient results.

Theorem 1 For stochastic switched nonlinear systems (1), let (A) hold, if there exist a group of Lyapunov functions $V_{i}(t, x) \in \mathcal{C}^{1,2}\left(R_{+} \times R^{n}\right)$ and positive constants $p, b_{i}, c_{i}, \gamma$, and $\eta \geq 1$, such that, for all $t \geq 0, x \in R^{n}$

$$
\begin{aligned}
& b_{i}|x|^{p} \leq V_{i}(t, x) \leq c_{i}|x|^{p} \\
& \mathcal{L} V_{i}(t, x) \leq \gamma \psi_{1}(t) V_{i}(t, x)
\end{aligned}
$$

and at each switching instant $t_{k},(k=1,2, \ldots)$,

$$
V_{\sigma\left(t_{k}\right)}\left(t_{k}, x\left(t_{k}\right)\right) \leq \eta V_{\sigma\left(t_{k-1}\right)}\left(t_{k}, x\left(t_{k}\right)\right)
$$

Then, for every $x_{0} \in R^{n}$, there exists a solution $x(t)=x\left(t, x_{0}\right)$ on $\left[t_{0}, \infty\right)$ to stochastic switched nonlinear system (1). Moreover, the system (1) is p-th moment $\psi^{\gamma}$ stable and

$$
E\left|x\left(t, x_{0}\right)\right|^{p} \leq \frac{c}{b}\left\|x_{0}\right\|^{p} \psi^{\gamma}(t)
$$

Proof Let $x(t)=x\left(t, x_{0}\right)$ is a solution of stochastic switched nonlinear system (1) and $h(t)=\psi^{-\gamma}(t) E\left|x\left(t, x_{0}\right)\right|^{p}$.

We can give switching signal $\sigma(t)$ and instant $t$ for arbitrary, and assume that $t_{k}$ is the last switching instant before $t$, i.e. there is no switching on the interval $\left[t_{k}, t\right)$. 
By condition (5), we obtain

$$
\begin{aligned}
& b_{\sigma\left(t_{k}\right)} \psi^{-\gamma}(t)\left|x\left(t, x_{0}\right)\right|^{p} \leq \psi^{-\gamma}(t) V_{\sigma\left(t_{k}\right)}(t, x(t)) \\
& =\psi^{-\gamma}\left(t_{k}\right) V_{\sigma\left(t_{k}\right)}\left(t_{k}, x\left(t_{k}\right)\right)+\int_{t_{k}}^{t} d\left[\psi^{-\gamma}(s) V_{\sigma\left(t_{k}\right)}(s, x(s))\right] \\
& =\psi^{-\gamma}\left(t_{k}\right) V_{\sigma\left(t_{k}\right)}\left(t_{k}, x\left(t_{k}\right)\right)+\int_{t_{k}}^{t} \psi^{-\gamma}(s)\left[-\gamma \psi_{1}(s) V_{\sigma\left(t_{k}\right)}(s, x(s))\right. \\
& \left.+\mathcal{L} V_{\sigma\left(t_{k}\right)}(s, x(s))\right] d s \\
& +\int_{t_{k}}^{t} \psi^{-\gamma}(s) \frac{\partial V_{\sigma\left(t_{k}\right)}(s, x(s))}{\partial x} g_{\sigma\left(t_{k}\right)}(s, x(s)) d w(s) \\
& \leq \eta \psi^{-\gamma}\left(t_{k-1}\right) V_{\sigma\left(t_{k-1}\right)}\left(t_{k}, x\left(t_{k}\right)\right)+\int_{t_{k}}^{t} \psi^{-\gamma}(s) \\
& {\left[-\gamma \psi_{1}(s) V_{\sigma\left(t_{k}\right)}(s, x(s))+\mathcal{L} V_{\sigma\left(t_{k}\right)}(s, x(s))\right] d s} \\
& +\int_{t_{k}}^{t} \psi^{-\gamma}(s) \frac{\partial V_{\sigma\left(t_{k}\right)}(s, x(s))}{\partial x} g_{\sigma\left(t_{k}\right)}(s, x(s)) d w(s) \\
& =\eta \psi^{-\gamma}\left(t_{k-1}\right) V_{\sigma\left(t_{k-1}\right)}\left(t_{k-1}, x\left(t_{k-1}\right)\right)+\int_{t_{k-1}}^{t} \psi^{-\gamma}(s) \\
& {\left[-\gamma \psi_{1}(s) V_{\sigma(s)}(s, x(s))+\mathcal{L} V_{\sigma(s)}(s, x(s))\right] d s} \\
& +\int_{t_{k-1}}^{t} \psi^{-\gamma}(s) \frac{\partial V_{\sigma(s)}(s, x(s))}{\partial x} g_{\sigma(s)}(s, x(s)) d w(s) \\
& \leq \eta^{k} \psi^{-\gamma}\left(t_{0}\right) V_{\sigma\left(t_{0}\right)}\left(t_{0}, x\left(t_{0}\right)\right)+\int_{t_{0}}^{t} \psi^{-\gamma}(s)\left[-\gamma \psi_{1}(s) V_{\sigma(s)}(s, x(s))\right. \\
& \left.+\mathcal{L} V_{\sigma(s)}(s, x(s))\right] d s \\
& +\int_{t_{0}}^{t} \psi^{-\gamma}(s) \frac{\partial V_{\sigma(s)}(s, x(s))}{\partial x} g_{\sigma(s)}(s, x(s)) d w(s)
\end{aligned}
$$

Let $t_{0}=0$, we obtain

$$
\begin{aligned}
b_{\sigma\left(t_{k}\right)} \psi^{-\gamma}(t)\left|x\left(t, x_{0}\right)\right|^{p} & \leq \eta^{k} V_{\sigma\left(t_{0}\right)}\left(0, x_{0}\right)+\int_{0}^{t} \psi^{-\gamma}(s)\left[-\gamma \psi_{1}(s) V_{\sigma(s)}(s, x(s))\right. \\
& \left.+\mathcal{L} V_{\sigma(s)}(s, x(s))\right] d s+\int_{0}^{t} \psi^{-\gamma}(s) \frac{\partial V_{\sigma(s)}(s, x(s))}{\partial x} g_{\sigma(s)}(s, x(s)) d w(s)
\end{aligned}
$$

By condition (5), we have

$$
\begin{aligned}
b_{\sigma\left(t_{k}\right)} \psi^{-\gamma}(t)\left|x\left(t, x_{0}\right)\right|^{p} \leq & \eta^{k} c_{\sigma\left(t_{k}\right)}\left|x_{0}\right|^{p}+\int_{0}^{t} \psi^{-\gamma}(s)\left[-\gamma \psi_{1}(s) V_{\sigma(s)}(s, x(s))\right. \\
& +\mathcal{L} V \sigma(s)(s, x(s))] d s+M(t)
\end{aligned}
$$

where $M(t)=\int_{0}^{t} \psi^{-\gamma}(s) \frac{\partial V_{\sigma(s)}(s, x(s))}{\partial x} g(s, x(s)) d w(s)$ is a continuous local martingale with initial value $M(0)=0$.

Substituting (6) into (9) results in

$$
b_{\sigma\left(t_{k}\right)} \psi^{-\gamma}(t)\left|x\left(t, x_{0}\right)\right|^{p} \leq \eta^{k} c_{\sigma\left(t_{k}\right)}\left|x_{0}\right|^{p}+M(t) .
$$


By Lemma 4, we have $b_{\sigma\left(t_{k}\right)} h(t) \leq \eta^{k} c_{\sigma\left(t_{k}\right)}\left|x_{0}\right|^{p}$. Then

$$
E\left|x\left(t, x_{0}\right)\right|^{p} \leq \frac{c_{\sigma\left(t_{k}\right)} \eta^{k}}{b_{\sigma\left(t_{k}\right)}}\left\|x_{0}\right\|^{p} \psi^{\gamma}(t)
$$

Let $b=\min \left\{b_{i}\left\{\right.\right.$ and $c=\max \left\{c_{i} \eta^{k}\right\}$, we obtain

$$
E\left|x\left(t, x_{0}\right)\right|^{p} \leq \frac{c}{b}\left\|x_{0}\right\|^{p} \psi^{\gamma}(t), \quad t \geq 0
$$

Thus, the system (1) is p-th moment $\psi^{\gamma}$ stable.

Remark 1 Here we generalize our research to stochastic switched nonlinear systems. The result shows how to derive some useful conditions for stochastic switched systems in terms of the multiple Lyapunov functions method.

In the following, the almost sure $\psi^{\gamma}$ stability of system (1) is presented.

Theorem 2 For stochastic switched nonlinear system (1), let (A) hold, if there exist positive constants $p, b_{i}, \gamma, \eta \geq 1$, and a group of Lyapunov functions $V_{i}(t, x) \in \mathcal{C}^{1,2}\left(R_{+} \times R^{n}\right)$, such that, for all $t \geq 0$ and $x \neq 0$

$$
\begin{aligned}
& b_{i}|x|^{p} \leq V_{i}(t, x) \\
& \mathcal{L} V_{i}(t, x) \leq \gamma \psi_{1}(t) V_{i}(t, x)
\end{aligned}
$$

and at each switching instant $t_{k},(k=1,2, \ldots)$,

$$
V_{\sigma\left(t_{k}\right)}\left(t_{k}, x\left(t_{k}\right)\right) \leq \eta V_{\sigma\left(t_{k-1}\right)}\left(t_{k}, x\left(t_{k}\right)\right)
$$

Then, for every $x_{0} \in R^{n}$, there exists a solution $x(t)=x\left(t, x_{0}\right)$ on $\left[t_{0}, \infty\right)$ to stochastic switched nonlinear system (1). Moreover, the system (1) is almost surely $\psi^{\gamma}$ stable and

$$
\limsup _{t \rightarrow \infty} \frac{\ln \left|x\left(t, x_{0}\right)\right|}{|\ln \psi(t)|} \leq-\frac{\gamma}{p} \quad \text { a.s.. }
$$

Proof Clearly, (13) holds for $x_{0}=0$ since $x\left(t, x_{0}\right) \equiv 0$. We therefore only need to show (13) for $x_{0} \neq 0$. Fix $x_{0} \neq 0$, let $x\left(t, x_{0}\right)=x(t)$ is a solution of stochastic switched nonlinear system (1). By condition (10), we have $\ln \left[b_{i}|x|^{p}\right] \leq \ln \left[V_{i}(t, x)\right]$.

According to Itô formula, we obtain

$$
d\left[\ln \left[V_{i}(t, x)\right]\right]=\frac{1}{V_{i}(t, x)}\left[\mathcal{L} V_{i}(t, x)-\frac{1}{2 V_{i}(t, x)}\left(\frac{\partial V_{i}(t, x)}{\partial x} g_{i}(t, x)\right)^{2} d t+\frac{\partial V_{i}(t, x)}{\partial x} g_{i}(t, x) d w(t)\right]
$$

where

$$
\mathcal{L} V_{i}(t, x)=\frac{\partial V_{i}(t, x)}{\partial t}+\frac{\partial V_{i}(t, x)}{\partial x} f_{i}(t, x)+\frac{1}{2} \operatorname{tr}\left[g_{i}^{T}(t, x) \frac{\partial^{2} V_{i}(t, x)}{\partial x^{2}} g_{i}(t, x)\right] .
$$


We can give switching signal $\sigma(t)$ and instant $t$ for arbitrary, and assume that $t_{k}$ is the last switching instant before $t$, i.e. there is no switching on the interval $\left[t_{k}, t\right)$.

By Itô formula, we have

$$
\begin{aligned}
\ln V_{\sigma\left(t_{k}\right)}(t, x(t))= & \ln V_{\sigma\left(t_{k}\right)}\left(t_{k}, x\left(t_{k}\right)\right)+\int_{t_{k}}^{t} \mathcal{L} \ln V_{\sigma\left(t_{k}\right)}(s, x(s)) d s \\
& +\int_{t_{k}}^{t} \frac{1}{V_{\sigma\left(t_{k}\right)}(s, x(s))} \frac{\partial V_{\sigma\left(t_{k}\right)}(s, x(s))}{\partial x} g_{\sigma\left(t_{k}\right)}(s, x(s)) d w(s) \\
\leq & \ln \left[\eta V_{\sigma\left(t_{k-1}\right)}\left(t_{k}, x\left(t_{k}\right)\right)\right]+\int_{t_{k}}^{t} \mathcal{L} \ln V_{\sigma\left(t_{k}\right)}(s, x(s)) d s \\
& +\int_{t_{k}}^{t} \frac{1}{V_{\sigma\left(t_{k}\right)}(s, x(s))} \frac{\partial V_{\sigma\left(t_{k}\right)}(s, x(s))}{\partial x} g_{\sigma\left(t_{k}\right)}(s, x(s)) d w(s) \\
= & \ln \left[\eta V_{\sigma\left(t_{k-1}\right)}\left(t_{k-1}, x\left(t_{k-1}\right)\right)\right]+\int_{t_{k-1}}^{t} \mathcal{L} \ln V_{\sigma(s)}(s, x(s)) d s \\
& +\int_{t_{k-1}}^{t} \frac{1}{V_{\sigma(s)}(s, x(s))} \frac{\partial V_{\sigma(s)}(s, x(s))}{\partial x} g_{\sigma(s)}(s, x(s)) d w(s) \\
\leq & \ln \left[\eta^{k} V_{\sigma\left(t_{0}\right)}\left(t_{0}, x\left(t_{0}\right)\right)\right]+\int_{t_{0}}^{t} \mathcal{L} \ln V_{\sigma(s)}(s, x(s)) d s \\
& +\int_{t_{0}}^{t} \frac{1}{V_{\sigma(s)}(s, x(s))} \frac{\partial V_{\sigma(s)}(s, x(s))}{\partial x} g_{\sigma(s)}(s, x(s)) d w(s)
\end{aligned}
$$

Let $t_{0}=0$, we obtain

$$
\begin{aligned}
\ln V_{\sigma\left(t_{k}\right)}(t, x(t)) \leq & \ln \left[\eta^{k} V_{\sigma\left(t_{0}\right)}\left(0, x_{0}\right)\right]+\int_{0}^{t} \frac{\mathcal{L} V_{\sigma(s)}(s, x(s))}{V_{\sigma(s)}(s, x(s))} d s \\
& -\frac{1}{2} \int_{0}^{t} \frac{1}{V_{\sigma(s)}^{2}(s, x(s))}\left|\frac{\partial V_{\sigma(s)}(s, x(s))}{\partial x} g_{\sigma(s)}(s, x(s))\right|^{2} d s+M(t)
\end{aligned}
$$

where $M(t)=\int_{0}^{t} \frac{1}{V_{\sigma(s)}(s, x(s))} \frac{\partial V_{\sigma(s)}(s, x(s))}{\partial x} g_{\sigma(s)}(s, x(s)) d w(s)$ is a continuous local martingale with initial value $M(0)=0$.

Substituting (11) into (14) results in

$$
\begin{aligned}
\ln V_{\sigma\left(t_{k}\right)}(t, x(t)) \leq & \ln \left[\eta^{k} V_{\sigma\left(t_{0}\right)}\left(0, x_{0}\right)\right]+\int_{0}^{t} \gamma \psi_{1}(s) d s \\
& -\frac{1}{2} \int_{0}^{t} \frac{1}{V_{\sigma(s)}^{2}(s, x(s))}\left|\frac{\partial V_{\sigma(s)}(s, x(s))}{\partial x} g_{\sigma(s)}(s, x(s))\right|^{2} d s+M(t) \\
= & \ln \left[\eta^{k} V_{\sigma\left(t_{0}\right)}\left(0, x_{0}\right)\right]+\gamma \ln \psi(t) \\
& -\frac{1}{2} \int_{0}^{t} \frac{1}{V_{\sigma(s)}^{2}(s, x(s))}\left|\frac{\partial V_{\sigma(s)}(s, x(s))}{\partial x} g_{\sigma(s)}(s, x(s))\right|^{2} d s+M(t)
\end{aligned}
$$

Therefore,

$$
\begin{aligned}
\left.\ln \left|b_{\sigma\left(t_{k}\right)}\right| x\right|^{p} \mid \leq & \ln \left[\eta^{k} V_{\sigma\left(t_{0}\right)}\left(0, x_{0}\right)\right]+\gamma \ln \psi(t) \\
& -\frac{1}{2} \int_{0}^{t} \frac{1}{V_{\sigma(s)}^{2}(s, x(s))}\left|\frac{\partial V_{\sigma(s)}(s, x(s))}{\partial x} g_{\sigma(s)}(s, x(s))\right|^{2} d s+M(t)
\end{aligned}
$$


Let $z(t)=\frac{1}{V_{\sigma(t)}(t, x(t))} \frac{\partial V_{\sigma(t)}(t, x(t))}{\partial x} g_{\sigma(t)}(t, x(t))$, by Lemma $6, \forall n \in N, \forall \delta>0$, we have

$$
P\left(\sup _{0 \leq t \leq n+1}\left[\frac{|\ln \psi(t)|}{t} M(t)-\frac{\delta}{2} \frac{|\ln \psi(t)|^{2}}{t^{2}} \int_{0}^{t}|z(s)|^{2} d s\right] \geq \frac{2 \ln n}{\delta}\right) \leq \frac{1}{n^{2}} .
$$

By Lemma 7, when $n \rightarrow \infty, n \leq t \leq n+1$, we obtain

$$
\frac{|\ln \psi(t)|}{t} M(t) \leq \frac{\delta}{2} \frac{|\ln \psi(t)|^{2}}{t^{2}} \int_{0}^{t}|z(s)|^{2} d s+\frac{2 \ln t}{\delta}, \quad \text { a.s. }
$$

That is

$$
M(t) \leq \delta \frac{|\ln \psi(t)|}{2 t} \int_{0}^{t}|z(s)|^{2} d s+\frac{2 t \ln t}{\delta|\ln \psi(t)|}, \quad \text { a.s. }
$$

Substituting (16) into (15) results in

$$
\begin{aligned}
& \ln \left(b_{\left.\sigma\left(t_{k}\right)|x|^{p}\right)} \leq \ln \left[\eta^{k} V_{\sigma\left(t_{0}\right)}\left(0, x_{0}\right)\right]+\gamma \ln \psi(t)-\left(\frac{1}{2}-\frac{\delta|\ln \psi(t)|}{2 t}\right) \int_{0}^{t}|z(s)|^{2} d s+\frac{2 t \ln t}{\delta|\ln \psi(t)|}\right. \\
& \leq \ln \left[\eta^{k} V_{\sigma\left(t_{0}\right)}\left(0, x_{0}\right)\right]+\gamma \ln \psi(t)-\left(\frac{1}{2}-\frac{\delta|\ln \psi(t)|}{2 t}\right) t \max _{0 \leq s \leq t}|z(s)|^{2}+\frac{2 t \ln t}{\delta|\ln \psi(t)|} \\
&=\ln \left[\eta^{k} V_{\sigma\left(t_{0}\right)}\left(0, x_{0}\right)\right]+\gamma \ln \psi(t)-\frac{t}{2} \max _{0 \leq s \leq t}|z(s)|^{2}+\frac{\delta}{2} \max _{0 \leq s \leq t}|z(s)|^{2}|\ln \psi(t)|+\frac{2 t \ln t}{\delta|\ln \psi(t)|}
\end{aligned}
$$

Then

$\frac{\ln \left(b_{\sigma\left(t_{k}\right)}|x|^{p}\right)}{|\ln \psi(t)|} \leq \frac{\ln \left[\eta^{k} V_{\sigma\left(t_{0}\right)}\left(0, x_{0}\right)\right]}{|\ln \psi(t)|}-\gamma-\frac{1}{2} \frac{t}{|\ln \psi(t)|} \max _{0 \leq s \leq t}|z(s)|^{2}+\frac{\delta}{2} \max _{0 \leq s \leq t}|z(s)|^{2}+\frac{2 t \ln t}{\delta|\ln \psi(t)|^{2}}$

Let $t \rightarrow \infty, \delta \rightarrow 0$, respectively. We have

$$
\limsup _{t \rightarrow \infty} \frac{\ln \left(b_{\sigma\left(t_{k}\right)}|x|^{p}\right)}{|\ln \psi(t)|} \leq-\gamma, \quad \text { a.s. }
$$

That is,

$$
\limsup _{t \rightarrow \infty} \frac{\ln \left|x\left(t, x_{0}\right)\right|}{|\ln \psi(t)|} \leq-\frac{\gamma}{p}, \quad \text { a.s. }
$$

Thus, the system (1) is almost surely $\psi^{\gamma}$ stable.

Remark 2 Compared to the Theorem 3.3 and 6.2 given in Mao (1997), Theorem 1 and 2 extend the stability results from stochastic nonlinear systems to stochastic switched nonlinear systems. The systems given in Mao (1997) can be thought as a special case of system (1). Moreover, in Mao (1994, 1997) Filipovic (2009), Cong et al. (2011) the authors studied exponential stability, in Theorem 1 and 2, we investigated $\psi^{\gamma}$ stability, the concept of $\psi^{\gamma}$ stability contains exponential stability and polynomial stability, it has a wide applicability. In addition, for deterministic case, the researchers considered ordinary differential equations, in this paper, we considered stochastic differential equations. 


\section{Examples}

In this section, a numerical example is given to illustrate the effectiveness of the main results established in "Main results" section as follows.

Consider the stochastic switched nonlinear system (1). where $\sigma(t):\left[t_{0}, \infty\right) \rightarrow\{1,2\}$ is the switching signal. Let $\left\{t_{1}<t_{2}<\cdots<t_{k}<\cdots\right\}$ be a switching sequence and the $i_{k}$ th subsystem is active at time interval $\left[t_{k}, t_{k+1}\right)$, where $t_{k}$ is the switching instant, $k=0,1,2, \cdots$ and $i_{k} \in\{1,2\}$.

We choose $t_{k+1}-t_{k}=0.6 s, \quad V_{1}(t, x)=V_{2}(t, x)=x^{2}, \quad \psi(t)=\frac{e^{-t}}{1+t}, t \geq 0, \quad$ then, $\psi(0)=1, \psi(t) \rightarrow 0 \quad$ as $\quad t \rightarrow \infty, \quad \psi^{\prime}(t)=-\frac{2+t}{(1+t)^{2}} e^{-t}, \quad \psi_{1}(t)=\frac{\psi^{\prime}(t)}{\psi(t)}=-\left(1+\frac{1}{1+t}\right)$, $-2 \leq \psi_{1}(t) \leq-1$.

When $\sigma(t)=1$, we choose $f_{1}(t, x)=-3 x$ and $g_{1}(t, x)=\sqrt{2} x$ for the first subsystem; when $\sigma(t)=2$, we choose $f_{2}(t, x)=-2 x-\operatorname{sgn} x$ and $g_{2}(t, x)=\sqrt{2|x|}$ for the second subsystem.

For the first subsystem, If $x \neq 0$, we have

$$
\begin{aligned}
\mathcal{L} V_{1}(t, x) & =2 x \cdot(-3 x)+\frac{1}{2} \cdot 2 \cdot 2 x^{2}=-4 x^{2} \\
& =-4 V_{1}(t, x) \leq 2 \psi_{1}(t) V_{1}(t, x)
\end{aligned}
$$

If $x=0$ then, $V_{1}(t, x)=0, \mathcal{L} V_{1}(t, x)=0$.

For the second subsystem, If $x \neq 0$, we have

$$
\begin{aligned}
\mathcal{L} V_{2}(t, x) & =2 x \cdot(-2 x-\operatorname{sgn} x)+\frac{1}{2} \cdot 2 \cdot 2 \cdot|x|=-4 x^{2} \\
& =-4 V_{2}(t, x) \leq 2 \psi_{1}(t) V_{2}(t, x)
\end{aligned}
$$

If $x=0$ then, $V_{2}(t, x)=0, \mathcal{L} V_{2}(t, x)=0$.

By Theorem 1, we can choose $p=2, \gamma=2$, then $\mathcal{L} V_{i}(t, x) \leq \gamma \psi_{1}(t) V_{i}(t, x), i=1,2$, which means that the conditions of Theorem 1 are satisfied. So the stochastic switched nonlinear systems are $\mathrm{p}$-th moment $\psi^{\gamma}$ stable. The switching signal and the state trajectory are presented in Figs. 1 and 2 respectively.

Remark 3 In this example, a stochastic switched nonlinear system is constructed to show the efficiency of the main results. Figure 1 describes switching signal changes over the time. Figure 2 depicts state trajectory changes over the time and shows the system is p-th moment $\psi^{\gamma}$ stability.

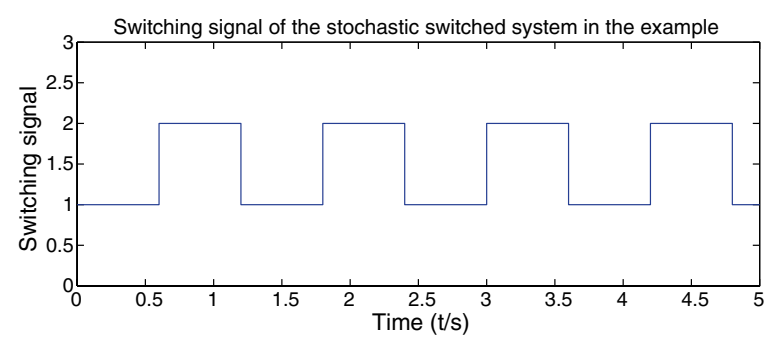

Fig. 1 Switching signal of the stochastic switched nonlinear systems 


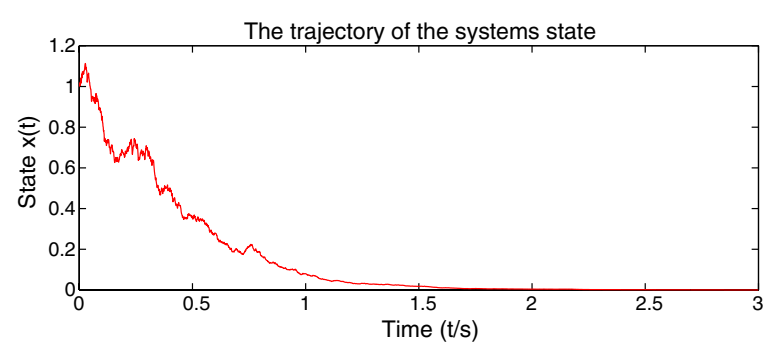

Fig. 2 The trajectory of the stochastic switched nonlinear systems state

\section{Conclusions}

In this paper, p-th moment and almost sure $\psi^{\gamma}$ stability have been investigated for a class of stochastic switched nonlinear systems. Some sufficient conditions have been derived to check the stability criteria by using multiple Lyapunov functions approach. A numerical example is provided to verify the effectiveness of the main results. Our future research will be focus on $\psi^{\gamma}$ stability of stochastic switched nonlinear systems with delay and $\psi^{\gamma}$ stability of neutral stochastic switched nonlinear systems with delay.

\section{Authors' contributions}

This work was carried out in collaboration among the authors. All authors have a good contribution to design the study, and to perform the analysis of this research work. All authors read and approved the final manuscript.

\section{Acknowledgements}

The work was supported by the National Natural Science Foundation of China under Grant No. 11261033, and the Postgraduate Scientific Research Innovation Foundation of Inner Mongolia under Grant No. 1402020201336.

\section{Competing interests}

The authors declare that they have no competing interests.

Received: 23 December 2015 Accepted: 14 November 2016

Published online: 24 November 2016

\section{References}

Ai Z, Zong G (2014) Finite-time stochastic input-to-state stability of impulsive switched stochastic nonlinear systems. Appl Math Comput 245:462-473

Aleksandrov A, Chen Y, Platonov A, Zhang L (2011) Stability analysis for a class of switched nonlinear systems. Automatica 47:2286-2291

Branicky M (1998) Multiple Lyapunov functions and other analysis tools for switched and hybrid systems. IEEE Trans Autom Control 43(4):475-482

Chatterjee D, Liberzon D (2006) Stability analysis of deterministic and stochastic switched systems via a comparison principle and multiple Lyapunov functions. SIAM J Control Optim 45:174-206

Chatterjee D, Liberzon D (2004) On stability of switched stochastic systems. In: 43rd IEEE conference on decision and control, pp 4125-4127

Cong S, Jiang H, Sheng Z (2011) Analysis on exponential stability for switched linear stochastic systems: multiple Lyapunov functions approach. J Eng Heilongjiang Univ 2:100-104

Dimarogonas D, Kyriakopoulos K (2004) Lyapunov-like stability of switched stochastic systems. In: Proceeding of the 2004 American control conference, pp 1868-1872

Feng W, Zhang J (2006) Stability analysis and stabilization control of multi-variable switched stochastic systems. Automatica 42:169-176

Feng W, Tian J, Zhao P (2011) Stability analysis of switched stochastic systems. Automatica 47:148-157

Filipovic V (2009) Exponential stability of stochastic switched systems. Trans Inst Meas Control 31:205-212

Goetz R, Hritonenko N (2008) The optimal economic lifetime of vintage capital in the presence of operating costs, technological progress and learning. J Econ Dyn Control 32:3032-3053

Hu B, Xu X, Michel A, Antsaklis P (1999) Stability analysis for a class of nonlinear switched systems. In: Proceedings of the 38th conference on decision \& control phoenix, Arizona, USA, pp 4374-4379

Hu S, Huang C, Wu F (2008) Stochastic differential equations. Science Press, Beijing

Khasminskii R (1980) Stochastic stability of differential equations. International Publisher, Rockville 
Krystul J (2001) Modeling of stochastic hybrid systems with applications to accident risk assessment. Ph.D. dissertation, University of Twente, Enschede

Mao X (1994) Exponential stability of stochastic differential equations. Marcel Dekker, New York

Mao X (1997) Stochastic differential equations and applications, 2nd edn. Horwood, Chichester

$\varnothing$ ksendal B (2005) Stochastic differential equations: an introduction with applications. Springer, Berlin

Pavlovic G, Jankovic S (2012) Razumikhin-type theorems on general decay stability of stochastic functional differential equations with infinite delay. J Comput Appl Math 236:1679-1690

Shen Y, Wang J (2009) Almost surely exponential stability of recurrent neural networks with Markovian switching. IEEE Trans Neural Netw 20(5):840-855

Wu F, Hu S (2012) Razumikhin-type theorems on general decay stability and robustness for stochastic functional differential equations. Int J Robust Nonlinear Control 22:763-777

Wu Z, Cui M, Shi P, Karimi H (2013) Stability of stochastic nonlinear systems with state-dependent switching. In: IEEE transactions on automatic control, pp 1904-1918

Xie G, Wang L (2003) Controllability and stabilizability of switched linear systems. Syst Control Lett 48(2):135-155

Zhang W, Chen B (2004) On stabilizability and exact observability of stochastic systems with their applications. Automatica 40:87-94

Zhang Q, Nie Z (2004) Exponential stability of stochastic age-dependent population dynamics system. Control Theory Appl 21(6):907-910

Zhang H, Wu Z, Xia Y (2014) Exponential stability of stochastic systems with hysteresis switching. Automatica 50:599-606

\section{Submit your manuscript to a SpringerOpen ${ }^{\circ}$ journal and benefit from:}

- Convenient online submission

\section{- Rigorous peer review}

- Immediate publication on acceptance

- Open access: articles freely available online

- High visibility within the field

- Retaining the copyright to your article

Submit your next manuscript at $\boldsymbol{\nabla}$ springeropen.com 